\title{
Axis Specific Player Load to Quantify Lower Limb Biomechanical Loading in Adolescent Badminton Players
}

\author{
Matthew James Wylde',2, Baranee Kumar', Low Chee Youngl and Andrew James \\ Callaway ${ }^{2}$ \\ I National Youth Sports Institute, Singapore \\ 2 Bournemouth University, Department of Rehabilitation and Sport Sciences, \\ Bournemouth, United Kingdom \\ E-mail: matthew_wylde@nysi.org.sg
}

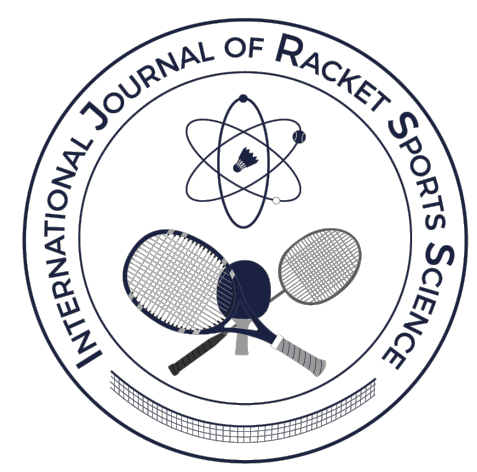

\begin{abstract}
While the use of accelerometer derived Player Load has become increasingly prominent, the limitation of this approach is that training load is reduced to a single number with no differentiation between the mechanisms of loading, resulting in a loss of context. As recovery from different loadings occur at different rates, the inability to differentiate between the loading mechanisms could lead to under or over training in one or more of these mechanisms. This study sought to compare axis specific accelerometer derived Player Load with differential RPE scores to establish a means of quantifying the lower limb biomechanical load of adolescent badminton training, to try and understand some of the context into the Player Load number. It was postulated that the Player Load from the vertical axis would provide a more precise measure of lower limb loading as other loading parameters, such as upper body rotation observed during a smash, would be removed from the calculation. Nineteen adolescent badminton players (Age: $14.0 \pm 0.8 \mathrm{y}$ ) based at a dedicated high performance youth training environment wore a GPS-embedded accelerometer between the scapulae in a purpose built vest during court-based training. After each training session the participants provided two RPE scores, one localised for the legs and one for breathlessness. Overall low correlations were observed between the Player Load and RPE values. The Player Load for the vertical axis showed a stronger correlation with the RPE for breathlessness than the RPE for the lower limb stress. The results from this study suggest that axis specific Player Load from the vertical axis does not provide greater insight into lower-limb biomechanical load compared to overall Player Load in adolescent badminton players.
\end{abstract}

Keywords: Badminton, Adolescent, Accelerometers, Training Load, RPE

Cite this article as:

Wylde, M., Kumar, B., Yong, L. C., \& Callaway, A. J. (2019). Axis Specific Player Load to Quantify Lower Limb Biomechanical Loading in Adolescent Badminton Players. International Journal of Racket Sports Science, 1(1), 37-44. 


\section{Introduction}

Monitoring the loading experienced by an athlete during training or competition is essential for determining whether the athlete is adapting to a training programme, understanding the need for recovery and reducing injury risks (Bourdon et al., 2017). While an optimal "dose" of load will create adaptations that will result in performance improvement, too little will blunt adaptations and too much will result in overuse injury and illness (Vanrenterghem, Nedergaard, Robinson, \& Drust, 2017). Furthermore, sudden spikes in load have been linked to injury occurrences across a range of sports (Gabbett, 2016). The need to manage loading in youth athletes is especially important as there is a growing body of evidence that has demonstrated an increase in overuse injuries amongst youth athletes and has linked inappropriate loads to injury and illness within this population (Murray, 2017). This evidence indicates that when dealing with youth athletes, planning appropriate loads and management of loading patterns is important to support a long sporting career (Bourdon et al., 2017).

The use of commercially available athlete tracking systems which incorporate GPS and inertial measurement units (IMUs) have become increasingly popular as a method of assessing athlete load. Catapult Innovations (Melbourne, Australia) developed a modified vector magnitude parameter called "Player Load" by integrating accumulated data from 3 accelerometers within the MinimaxX units (Boyd, Ball, \& Aughey, 2011). In this context, Player Load is therefore the summed multidirectional acceleration and deceleration of a player's movements throughout a session. The Player Load calculation has been used in indoor court based sports where the use of GPS is not possible and the cost of local positioning systems (LPS) is prohibitively expensive (Cormack, Smith, Mooney, Yong, \& O'Brien, 2013).

Player Load has been compared to internal load measures derived from heart-rate during badminton play (Abdullahi, Coetzee, \& Van Dan Berg, 2019). This study found that Player Load was only correlated to the heart-rate measures at the high intensity zone but not at the low or medium intensity zones, with the latter showing a negative correlation. The authors concluded that while the high intensity movements in badminton, for example an overhead smash, would elicit a clear heart-rate response, $183.5 \pm 5$ beats.min-1 (Ghosh, 2008), the overall high work density observed in badminton compared to field based sports made it difficult to observe clear differences in the low and medium intensity zones. The limitation with this approach is that Player Load is reported as a single number with no way of differentiating how this load was accumulated. While "relative distance" was also reported, this metric equates the Player Load to a distance covered on a running track, an approach which may not be suitable for a court-based sport such as badminton.

Understanding how load is accumulated is important, as adaptations from different forms of loading occur in different timeframes. For example, recovery from physiological loading may take only a few hours for a well-trained athlete, while recovery from biomechanical loading may take a few days. A framework for differentiating between the physiological and biomechanical load was conceptualised by Vanrenterghem et al. (2017). The danger would occur when an athlete returns to training when recovered from the physiological load but under recovered from the biomechanical load, which may result in overuse injury. Conversely, if an athlete only continues physiological loading when fully recovered from the biomechanical load, the physiological system may be undertrained which would result in a performance decrement.

Within a youth population the management of biomechanical load is of particular importance as youth athletes are still developing fundamental movement skills and muscular strength. For example, a study of youth soccer players found that occurrences of knee valgus decreased with age and physical maturity (Read, Oliver, De Ste Croix, Myer, \& Lloyd, 2018). In a badminton context, $64 \%$ of injuries recorded in youth players were soft-tissue sprains and strains with knee injuries being the most common, accounting for $42 \%$ of injuries to the lower limbs (Goh, Mokhtar, \& Mohamad Ali, 2013). With this context, the 
measurement of lower limb biomechanical load would be essential in the prescription of optimal loading strategies for youth badminton players.

While the majority of studies report Player Load as a single score, Fish and Grieg (2014) reported in netball match-play the load separately for each of the acceleration axes. A similar approach may provide greater clarity as to how load is accumulated by youth Badminton players. Player Load from the vertical axis may provide a more precise measure of lower limb loading by removing other loading variables such as upper body rotation observed during a smash. Therefore, the purpose of this study was to evaluate whether Player Load from the vertical axis provides a more precise measurement of lower limb loading as compared to total Player Load or the Player Load from the antero-posterior and medio-lateral axis.

\section{Method}

The participants for this study were 19 adolescent badminton players (age: $14.0 \pm 0.8$ years) based at a dedicated high performance youth training environment. The student-athletes were assessed over a 4-week period within which they would train twice a day from Monday to Friday and once a day on Saturday. Only court based training was assessed and gym based training was excluded. Each student-athlete wore a VX Sport (Visuallex Sport International, Lower Hutt, New Zealand) data logging unit (dimensions: $74 \mathrm{~mm} \times 47$ $\mathrm{mm} \times 17 \mathrm{~mm}$; mass: $50 \mathrm{~g}$ ) between the scapulae in a purpose built harness during each court-based training session for the duration of the data collection. The VX Sport system has been found to possess both high intra-system and inter-system reliability with the Catapult Optimeye S5 system (Wylde, Lee, Low, \& Callaway, 2018). However, to further limit any interunit reliability issues, the student-athletes wore the same unit throughout the assessment period. After each training session the student-athletes provided two rating of perceived exertion (RPE) scores (between 1 and 10, with 1 being low exertion), "RPE-L" being RPE localised for the legs and RPE-B being a rating for breathlessness (Weston, Siegler, Bahnert, McBrien, \& Lovell, 2015). Prior to the data collections the studentathletes were briefed on the process and how to differentiate between the two RPEs, while pictures of lungs and legs were used in the record sheet to aide understanding (Figure 1).

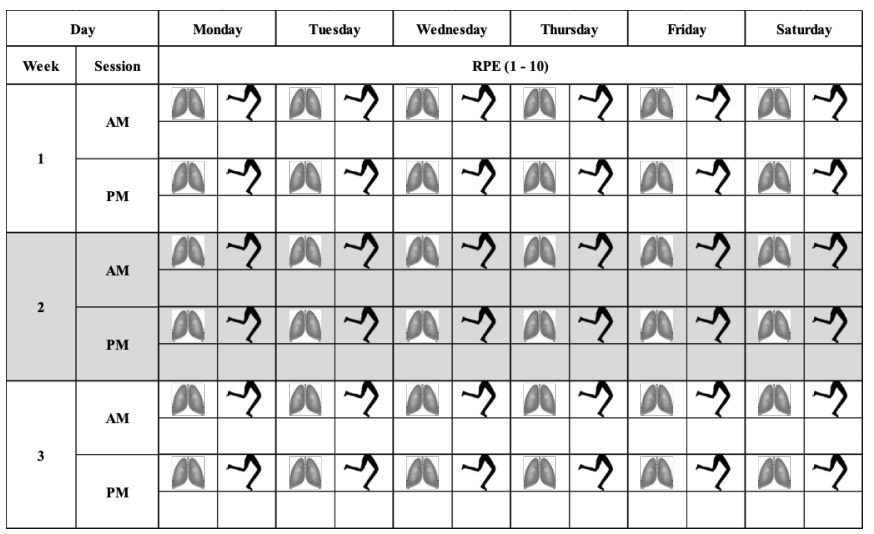

Figure 1. Differential RPE record sheet

After the completion of each training day, the accelerometer data were extracted at $100 \mathrm{~Hz}$ using the accompanying VX Sport software. The raw data was filtered at $10 \mathrm{~Hz}$ using a 3rd order Butterworth filter and centred mean in Matlab (MathWorks, Natick, MA, USA). The Player Load was calculated using a modified vector magnitude calculation, being the square root of the sum of activity counts squared (Boyd et al., 2011) (Equation 1) and the load for the vertical, anteroposterior and medio-lateral axis were also calculated (Equation 2).

\section{Player Load}

$=\sqrt{\frac{\left(a x_{1}-a x_{-1}\right)^{2}+\left(a y_{1}-a y_{-1}\right)^{2}+\left(a z_{1}-a z_{-1}\right)^{2}}{100}}$ Equation 1. Total (Vector Magnitude) Player Load. Where $\mathrm{a}=$ accelerometer value; $\mathrm{x}, \mathrm{y}, \mathrm{z}$ represents the medio-lateral, anterio-posterior, and vertical axes respectively. The units of measurement are reported as arbitrary units (AU).

$$
\text { Vertical Load }=\sqrt{\frac{\left(a z_{1}-a z_{-1}\right)^{2}}{100}}
$$




$$
\begin{gathered}
\text { Antero - Posterior Load }=\sqrt{\frac{\left(a y_{1}-a y_{-1}\right)^{2}}{100}} \\
\text { Medio - Lateral Load }=\sqrt{\frac{\left(a x_{1}-a x_{-1}\right)^{2}}{100}}
\end{gathered}
$$

Equation 2. Vertical, antero-posterior and mediolateral load calculations.

To assess the sensitivity of the measures to differentiate between players of different capability, the players were split into two groups based on chronological age, "Lower Secondary" (aged 12 to 14 years) and "Upper Secondary" (aged 14 to 16 years).
Cohen's Effect Sizes (Cohen, 1988) with modified descriptors (Hopkins, 2000) were used to assess the difference between the two groups. Pearson's correlation coefficient was used to assess the relationship between the various RPE scores and Player Load. Statistical computations were performed using SPSS v.24 (IBM Corp, Armonk, NY, USA) and statistical significance was accepted at $\mathrm{p}<0.05$.

\section{Results}

The descriptive data from the training sessions are outlined in Table 1. In general, there were "trivial" and "small" differences observed between the Lower Secondary and Upper Secondary groups. The only "moderate" effect size difference was observed for the Antero-Posterior Load and the RPE-L measures.

Table 1.

\begin{tabular}{|c|c|c|c|c|}
\hline Measure & $\begin{array}{l}\text { All Age Groups } \\
\qquad(\mathrm{n}=218) \\
\text { Mean } \pm S D\end{array}$ & $\begin{array}{l}\text { Lower Secondary } \\
\qquad \begin{array}{c}(\mathrm{n}=85) \\
\text { Mean } \pm S D\end{array}\end{array}$ & $\begin{array}{l}\text { Upper Secondary } \\
\qquad \begin{array}{c}(\mathrm{n}=133) \\
\text { Mean } \pm S D\end{array}\end{array}$ & $\begin{array}{c}\text { Effect Size } \\
\text { Lower Sec vs. Upper } \\
\text { Sec }\end{array}$ \\
\hline Duration (min) & $113.83 \pm 39.08$ & $112.48 \pm 40.43$ & $114.69 \pm 38.33$ & Trivial \\
\hline Total Load (AU) & $1678.91 \pm 700.01$ & $1441.19 \pm 552.38$ & $1830.83 \pm 742.79$ & Small \\
\hline Vertical Load (AU) & $989.92 \pm 442.84$ & $863.67 \pm 356.27$ & $1070.6 \pm 474.13$ & Small \\
\hline $\begin{array}{l}\text { Antero-Posterior Load } \\
\text { (AU) }\end{array}$ & $815.04 \pm 372.97$ & $680.24 \pm 310.3$ & $901.19 \pm 385.02$ & Moderate \\
\hline $\begin{array}{l}\text { Medio-Lateral Load } \\
(\mathrm{AU})\end{array}$ & $713.55 \pm 305.62$ & $610 \pm 228.42$ & $779.73 \pm 330.19$ & Small \\
\hline RPE-L (AU) & $6.83 \pm 1.55$ & $7.41 \pm 1.31$ & $6.47 \pm 1.57$ & Moderate \\
\hline RPE-B (AU) & $6.53 \pm 1.63$ & $7.08 \pm 1.3$ & $6.18 \pm 1.73$ & Small \\
\hline
\end{tabular}

Descriptive training load data per training session. $A U=$ arbitrary units.

The correlations were all found to be significant at $\mathrm{p}<0.05$ (Table 2). Stronger correlations were observed when both the Lower Secondary and Upper Secondary groups were viewed in isolation. In the Lower Secondary group, the strongest correlation was observed between Vertical Load and RPE-L, while for the Upper Secondary group the strongest correlation was observed between Total Load and RPE-B. 
Table 2.

Pearson correlation coefficient between Player Load and differential RPEs

\begin{tabular}{lcccc}
\hline & Total Load & Vertical Load & $\begin{array}{c}\text { Antero-Posterior } \\
\text { Load }\end{array}$ & $\begin{array}{c}\text { Medio-Lateral } \\
\text { Load }\end{array}$ \\
\hline All Age Groups $(\mathrm{n}=218)$ & & & & \\
\hline RPE-L & $0.126^{*}$ & $0.134^{*}$ & 0.095 & 0.086 \\
RPE-B & $0.180^{* *}$ & $0.182^{* *}$ & $0.159^{*}$ & $0.121^{*}$ \\
\hline Lower Secondary $(\mathrm{n}=85)$ & & & $0.185^{*}$ \\
\hline RPE-L & $0.235^{*}$ & $0.244^{*}$ & $0.200^{*}$ & 0.035 \\
RPE-B & 0.163 & $0.191^{*}$ & 0.164 & \\
\hline Upper Secondary $(\mathrm{n}=133)$ & & & $0.182^{*}$ \\
RPE-L & $0.223^{* *}$ & $0.208^{*}$ & $0.199^{*}$ & $0.268^{* *}$ \\
\hline
\end{tabular}

* Significance of $<0.5 \quad * *$ Significance of $<0.05$

\section{Discussion}

The correlations between Player Load and differential RPE, although significant, were low which is consistent with the findings from the study of Australian Football, where "trivial", "small" or "unclear" were observed between the player load values and the differential RPEs (Weston et al., 2015). In this study the RPE-B value, which represented the participants' perceived breathlessness, were more highly correlated to the Player Load compared to the RPE-L, which represented the lower limb biomechanical load. Contrary to the expectations of this study, the Vertical Load was more strongly correlated with the RPE-B and not RPE-L.

While session RPE has been shown to be a valid form of quantifying training load in youth athletes (Haddad et al., 2011; Padulo et al., 2014), it has been observed that youth athletes with greater training experience are able to more accurately perceive exertion compared to youth athletes with less experience (Barroso, Cardoso, Carmo, \& Tricolo, 2014). Therefore, it was assumed that the older group (Upper Secondary), with a longer training history, would provide more reliable RPE scores compared to the younger and less experienced group (Lower Secondary). In this study, the Upper Secondary group demonstrated a stronger correlation between the Player Load and the RPE-B values, while in contrast the Lower Secondary group recorded stronger correlations between the Player Load and RPE-L values. The Lower Secondary group was the only instance where the strongest correlation was between the Vertical Load and the RPE-L values.

While the use of RPE to quantify training load has been validated in tennis (Gomes, Moreira, Lodo, Capitani, \& Aoki, 2015), a study of elite junior tennis players highlighted the complexity of load perception (Murphy and Reid, 2013). In this study, the session RPE and drill RPE of junior tennis players during training were compared to the expected session RPE and drill RPE as rated by their coaches. While there were high levels of agreement between actual and expected drill RPE, there were significant differences between the actual and expected session RPE. This study highlighted that for junior tennis players the total session RPE is greater than the sum of the RPE of the individual drills. In a badminton context, explosive lower limb movements observed during training (jumps, lunges etc.) would create high Vertical Load and high RPE-L values. By contrast, holding a low position (isometric squat) while waiting for an opponent's shot, would produce low Vertical Load but potentially high RPE-L values. These "low load, high RPE" movements may explain the difference between the Vertical Load and RPE-L values found in the current study, as the total lower limb exertion of the session (RPE-L) is greater than the sum of the explosive lower limb movements (Vertical Load) within the session. 
The reporting of loads from the individual axis is currently not common place and the results from this study suggest that this approach may not provide any greater resolution to differentiate between lower limb and other types of loading for youth badminton players. In badminton match play, the lunge accounts for $15 \%$ of movements and produces high forces experienced in the lower limbs (Kuntze, Mansfield, $\&$ Sellers, 2009). Youth athletes have been shown to be inefficient in utilising the impact forces of the lunging movement in a Squash context (Williams and Kuitunen, 2010) emphasising the importance of understanding the loading associated with this movement. In a lunging movement the upper body does not remain upright meaning that the vertical axis of the accelerometer, when placed between the scapulae, is no longer aligned to the direction of the vertical force.

A novel approach has been devised for measuring loading of overhead strokes in badminton, combining video-based time-motion analysis and accelerometry (Saski, Nagano, \& Ichikawa, 2018). In this approach, movements with a load of greater than $4 \mathrm{~g}$ were isolated and manually classified based on the video of the movement. While this approach provided insights into the loading of single leg landings during overhead strokes, the authors acknowledged the arbitrary nature of the $4 \mathrm{~g}$ cut-off. In addition, the type of video-based time-motion analysis used in this study has been found to be labour intensive (Dobson and Keogh, 2007) and time-consuming (Jarning, Mok, Hansen, \& Bahr, 2015), meaning that it may not feasible to use this approach for monitoring of load in daily training for a large group of athletes.

Only readings from the accelerometer are used in the calculation of Player Load, the orientation of the unit in relation to the athlete during movement is not accounted for. This is not an issue when reporting total Player Load as data from all axis are combined during the calculation but becomes apparent when looking at the load for each axis in isolation. Combining readings from the accelerometer and gyroscope within the IMU may provide greater resolution regarding the type of loading being experienced. In Cricket fast bowling, McNamara,
Gabbet, Chapman, Naughton, \& Farhart (2015) were able to use measures from the accelerometer and gyroscope to differentiate between bowling and nonbowling actions. In addition, the application machine learning in a sport context is increasing able to identify specific movements using data derived from IMUs (Crust, Sweeting, Ball, \& Robertson, 2018). While such a machine learning approach has been used in badminton (Anand, Sharma, Srivastava, Kaligounder, \& Prakash, 2017), this was from using two wrist worn IMUs to identify stroke type (serve, clear, drop or smash). Further research is required to understand if these approaches could be applied in badminton to highlight movements, such as lunges and smashes, using a single trunk mounted IMU and then calculate the load generated by these movements. Such an approach would provide greater resolution and may provide an improved solution for measuring lower-limb loading in badminton.

\section{Conclusion}

This study sought to use differential Player Load scores and RPE to quantify lower limb load in the adolescent badminton players. Significant but low correlations were found between the Player Load and the differential RPEs. The Vertical Load did not provide any greater insight into player loading than the total Player Load variable. When the participants were split based on chorological age, both the Player Load and the Vertical Load for the younger players was more strongly correlated to the RPE-1 score while for older players they were more strongly correlated to the RPE-B score.

It is suggested that the reasons for these findings are that "low load, high RPE" movements (such as the isometric squat) are not well represented by the Player Load calculation and the vertical axis of the accelerometer is not aligned to the direction of the Vertical Load during key movements, such as lunges and smashes. As such, this does not provide of true representation of the Vertical Load created during these badminton specific movement. It is therefore proposed that a machine learning approach, which utilises both the accelerometer and gyroscope data from a single trunk mounted IMU, may provide an 
improved solution to attribute load to difference types of badminton specific movement and warrants further investigation.

\section{References}

Abdullahi, Y., Coetzee, B., \& Van Dan Berg, L. (2019). Relationships between results of an internal and external match load determining method in male, singles badminton players. Journal of Strength and Conditioning Research, 33(4), 1111-1118.

Anand, A., Sharma, M., Srivastava, R., Kaligounder, L., \& Prakash, D. (2017). Wearable motion sensor based analysis of swing sports. In $20171^{\text {th }}$ IEEE International Conference on Machine Learning and Applications (ICMLA), (p. 261-267). IEEE.

Barroso, R., Cardoso, R. K., Carmo, E. C., \& Tricolo., V. (2014). Perceived exertion in coaches and young swimmers with different training experience. International Journal of Sports Physiology and Performance, 9(2), 212-216.

Bourdon, P. C., Cardinale, M., Murray, A., Gast, P., Kellmann, M., Varley, M. C., Gabbett, T. J., Coutts, A. J., Burgess, D. J., Gregson, W., \& Cable, N. T. (2017). Monitoring athlete training loads: Consensus statement. International Journal of Sports Physiology and Performance, 12, S2. 161-170.

Boyd, L. J., Ball, K., \& Aughey, R. J. (2011). The reliability of MinimaxX accelrometers for measuring physical activity in Australian Football. International Journal of Sports Physiology and Performance, 6(3), 311321.

Cohen, J. (1988). Statistical Power Analysis for the Behavioral Sciences (2 ${ }^{\text {nd }}$ ed.). New Jersey: Lawrence Erlbaum.

Cormack, S. J., Smith, R. L., Mooney, M. M., Yong, W. B., \& O'Brien, B. J. (2013). Accelerometer load as a measure of activity profile in different standards of netball match play. International Journal of Sports Physiology and Performance, 9, 283-292.

Crust, E. E., Sweeting, A. J., Ball, K., \& Robertson, S. (2018). Machine and deep learning for sportspecific movement recognition: a systematic review of model development and performance. Journal of Sports Sciences, 37, 568-600.
Dobson, B. P., \& Keogh, J. W. L. (2007). Methodological issues for the application of timemotion analysis research. Strength and Conditioning Journal, 29, 48-55.

Fish, K., \& Greig, M. (2014). The influence of playing position on the biomechanical demands of Netball match-play. Journal of Athletic Enhancement, 3: 5.

Gabbett, T. J. (2016). The training-injury prevention paradox: should athletes be training harder and smarter? British Journal of Sports Medicine, 50(5), 273-280.

Ghosh, A. K. (2008). Heart rate and blood lactate responses during execution of some specifc strokes in badminton drills. International Journal of Applied Sports Science, 20(2), 27-36.

Goh S. L., Mokhtar A. H., \& Mohamad Ali M. R. (2013). Badminton injuries in youth competitive players. The Journal of Sports Medicine and Physical Fitness, 53(1), 65-70

Gomes, R. V., Moreira, A., Lodo. L., Capitani, C. D., \& Aoki, M. S. (2015). Ecological validity of session RPE method for quantifying internal training load in tennis. International Journal of Sports Science \& Coaching, 10(4), 729-737.

Haddad, M., Chaouachi, A., Castagna, C., Wong, D. P., Behm., D. G., \& Chamari, K. (2011). The construct validity of session RPE during an intensive camp in young male taekwondo athletes. International Journal of Sports Physiology and Performance, 6(2), 252263.

Hopkins, W. G. (2000). Measures of reliability in sports medicine and science. Sports Medicine, 30, 1-15.

Jarning, J. M., Mok, K. M., Hansen, B. H., \& Bahr, R. (2015). Application of a tri-axial accelerometer to estimate jump frequency in volleyball. Sports Biomechanics, 14, 95-105.

Kuntze, G., Mansfield, N., \& Sellers, W. (2010). A biomechanical analysis of common lunge tasks in badminton. Journal of Sports Sciences, 28(2), 183-191.

McNamara, D. J., Gabbett, T. J., Chapman, P., Naughton, G., \& Farhart, P. (2015). The validity of mirosensors to automatically detec bowling events and counts in Cricket fast bowlers. International Journal of Sports Physiology and Performance, 10, 71-75. 
Murphy, A., \& Reid, M. (2013). Comparison of athlete-coach perceptions of internal and external load markers for elite junior tennis training. International Journal of Sports Physiology and Performance, 9(5), 751-756.

Murray, A. (2017). Managing training load in adolescent athletes. International Journal of Sports Physiology and Performance, 12, S2.42-49.

Padulo, J., Chaabene, H., Tabben, M., Hadded, M., Gevat, C., Vando, S., Maurino, L., Chaouachi, A., \& Chamari, K. (2014). The construct validity of session RPE during an intensive camp in young male Karate athletes. Muscle, Ligaments and Tendons Journal, 4(2), 121-126.

Read, P. J., Oliver, J., De Ste Croix, M. B. A., Myer, G. D., \& Lloyd, R. S. (2018). Landing kinematics in elite male youth soccer players of different chronologic ages and stages of maturation. Journal of Athletic Training, 53(4), 372-378.

Sasaki, S., Nagano, Y., \& Ichikawa, H. (2018). Loading differences in single-leg landing in the forehand- and backhand-side courts after an overhead stroke in badminton: A novel tri-axial accelerometer research. Journal of Sports Sciences, 36(24), 2794-2801.

Vanrenterhgem, J., Nedergaard, N. J., Robinson, M. A., \& Drust, B. (2017). Training load monitoring in team sports: A novel framework separating physiological and biomechanical load-adaptation pathways. Sports Med, 47(11), 2135-2142.

Weston, M., Siegler, J., Bahnert, A., McBrien, J., \& Lovell, R. (2015). The application of differential ratings of perceived exertion to Australian Football League matches. Journal of Science and Medicine in Sport, 18, 704-708.

Williams, B. K., \& Kuitunen, S. (2010). Lunge forces and technique of junior squash players. Unpublished paper presented at the $28^{\text {th }}$ International Conference on Biomechanics in Sports, Michigan, USA, July.

Wylde, M. J., Lee, M. B. C., Low, C. Y., \& Callaway, A. J. (2018). Reliability and validity of GPSembedded accelerometers for the measurement of badminton specific player load. Journal of Trainology, 7(2), 34-37. 